\title{
THE GENERALIZED QUASILINEARIZATION METHOD FOR PARABOLIC INTEGRO-DIFFERENTIAL EQUATIONS
}

BY

A. S. VATSALA (Dept. of Mathematics, University of Louisiana at Lafayette, Lafayette, LA) AND

LIWEN WANG (Dept. of Mathematics, University of Louisiana at Lafayette, Lafayette, LA)

\begin{abstract}
In this paper we consider the nonlinear parabolic integro-differential equation with initial and boundary conditions. We develop the method of generalized quasilinearization to generate linear iterates that converge quadratically to the unique solution of the nonlinear parabolic integro-differential equation. For this purpose, we establish comparison results for the parabolic integro-differential equation. These comparison results are used to develop monotone sequences and to establish quadratic convergence.
\end{abstract}

1. Introduction. The method of quasilinearization due to Bellman [1] and Bellman and Kalaba [2] yields monotone sequences that converge quadratically to the unique solution of the nonlinear problem on the interval of existence. Further, the sequences are either increasing or decreasing depending on the forcing function being convex or concave. Recently the method of quasilinearization has been extended to a variety of nonlinear problems to obtain simultaneous bounds for the solution when the forcing function is neither convex nor concave. Yet, it has all the advantages of the method of quasilinearization. This method is now referred to as the generalized quasilinearization method. The generalized quasilinearization method is well developed for a variety of ordinary differential equations in [7]. The method of generalized quasilinearization has been developed to ordinary integro-differential equations in [9]. In this paper we develop the method of generalized quasilinearization for the nonlinear parabolic integrodifferential equations of Volterra type. The model we consider is a more general form of the Hodgkin-Huxley model for the propagation of the voltage pulse through a nerve axon, which is often referred to as the Fitzhugh-Nagumo equation. See [8] for more details. Here, we prove that the monotone sequences, which are solutions of linear parabolic integro-differential equations, converge quadratically to the unique solution of the nonlinear parabolic integro-differential equations. The quadratic convergence is achieved by developing a comparison theorem of the parabolic integro-differential equations with the

Received May 12, 1999.

2000 Mathematics Subject Classification. Primary 35B99, 45K05, 34K07. 
ordinary integro-differential equations. We note that the method of generalized quasilinearization provides a numerical procedure for the computation of solutions of nonlinear problems. Finally, we present a numerical example to demonstrate the application of our results.

2. Comparison theorem and main results. We consider the following problem:

$$
\begin{gathered}
L u=f(t, x, u(t, x))+\int_{0}^{t} g(t, x, s, u(s, x)) d s, \quad 0 \leq t \leq T, x \in \Omega, \\
u(0, x)=u_{0}(x), \quad x \in \Omega, \\
\left.u(t, x)\right|_{x \in \partial \Omega}=h(t, x),
\end{gathered}
$$

where $\Omega$ is a bounded open domain in $\mathbb{R}^{n}, L=\frac{\partial}{\partial t}-A$ is a parabolic operator, and

$$
A=\sum_{i, j=1}^{N} a_{i, j}(t, x) \frac{\partial^{2}}{\partial x_{i} \partial x_{j}}+\sum_{i=1}^{N} b_{i}(t, x) \frac{\partial}{\partial x_{i}}+c(t, x),
$$

with $a_{i, j}, b_{i}, c \in C^{\alpha, \alpha / 2}\left(\bar{Q}_{T}\right)$ and $\sigma_{0}|\xi|^{2} \leq \sum_{i, j=1}^{N} a_{i, j} \xi_{i} \xi_{j} \leq \sigma_{1}|\xi|^{2}, \sigma_{0}, \sigma_{1}>0$ for $(t, x) \in$ $\bar{Q}_{T}$ and $\xi \in \mathbb{R}^{n}$.

Throughout this paper we assume the initial and boundary conditions are such that $u_{0} \in C^{2+\alpha}(\bar{\Omega}), h \in C^{2+\alpha, 1+\alpha / 2}((0, T) \times \partial \Omega)$ and

$$
u_{0}(x)=h(0, x), \quad h_{t}=A u_{0}+f\left(0, x, u_{0}\right) \quad \text { for } t=0 \text { and } x \in \partial \Omega .
$$

Next we develop two comparison results, which we need in order to develop our main results. The first lemma will be used to show that the sequences we develop are monotone sequences. The second lemma will be used to prove that sequences converge quadratically to the unique solution of $(2.1)$.

LEMma 2.1. Assume that

(i) $g(t, x, s, u)$ is monotone nondecreasing in $u$ for each $(t, x, s)$ and

$$
\begin{aligned}
f(t, x, u)-f(t, x, v) & \leq M_{1}(u-v), \\
g(t, x, s, u)-g(t, x, s, v) & \leq M_{2}(u-v) \text { for } u \geq v,
\end{aligned}
$$

where $M_{i}$, for $i=1,2$, are nonnegative constants;

(ii) $v(t, x)$ and $w(t, x)$ satisfy

$$
\begin{aligned}
& L v \leq f(t, x, v(t, x))+\int_{0}^{t} g(t, x, s, v(s, x)) d s \\
& L w \geq f(t, x, w(t, x))+\int_{0}^{t} g(t, x, s, w(s, x)) d s,
\end{aligned}
$$

and

$$
\begin{aligned}
v(0, x) & \leq w(0, x), \\
\left.v(t, x)\right|_{x \in \partial \Omega} & \leq\left. w(t, x)\right|_{x \in \partial \Omega} .
\end{aligned}
$$

Then we have $w(t, x) \geq v(t, x)$. 
Proof. We shall first prove the result for strict inequalities. Assume that the conclusion of the lemma is false. Then there exists a point $\left(t_{0}, x_{0}\right)$ such that $w(t, x)>v(t, x)$ for $0 \leq t<t_{0}$, and $w\left(t_{0}, x_{0}\right)=v\left(t_{0}, x_{0}\right)$. Certainly $\left(t_{0}, x_{0}\right) \notin \partial \Omega$. Hence

$$
\begin{aligned}
L v\left(t_{0}, x_{0}\right) & <f\left(t_{0}, x_{0}, v\left(t_{0}, x_{0}\right)\right)+\int_{0}^{t_{0}} g\left(t, x_{0}, s, v\left(s, x_{0}\right)\right) d s \\
& \leq f\left(t_{0}, x_{0}, w\left(t_{0}, x_{0}\right)\right)+\int_{0}^{t_{0}} g\left(t, x_{0}, s, w\left(s, x_{0}\right)\right) d s \\
& <L w\left(t_{0}, x_{0}\right),
\end{aligned}
$$

i.e., $L\left[w\left(t_{0}, x_{0}\right)-v\left(t_{0}, x_{0}\right)\right]>0$.

If $\left(t_{0}, x_{0}\right) \in \Omega$, since $w(t, x)-v(t, x)$ has a minimum value at $\left(t_{0}, x_{0}\right)$, we have

$$
L\left[w\left(t_{0}, x_{0}\right)-v\left(t_{0}, x_{0}\right)\right] \leq 0,
$$

which leads to a contradiction. Therefore, we have $w(t, x)>v(t, x)$ for $t \geq 0$. In order to prove the result for nonstrict inequality, set

$$
w_{\varepsilon}(t, x)=w(t, x)+\varepsilon \exp (2 M t),
$$

where $\varepsilon>0$ and $M \geq \max \left\{\sqrt{M_{2} / 2}, M_{1}\right\}$. We have $v(0, x) \leq w(0, x)<w_{\varepsilon}(0, x)$. Since

$$
\begin{gathered}
f\left(t, x, w_{\varepsilon}(t, x)\right)-f(t, x, w(t, x)) \leq M_{1}\left(w_{\varepsilon}(t, x)-w(t, x)\right) \leq M \varepsilon \exp (2 M t), \\
\int_{0}^{t} g\left(t, x, s, w_{\varepsilon}(s, x)\right)-g(t, x, s, w(s, x)) d s \leq \frac{M_{2}}{2 M} \varepsilon(\exp (2 M t)-1)<M \varepsilon \exp (2 M t),
\end{gathered}
$$

we have

$$
\begin{aligned}
L w_{\varepsilon} & =L w+2 M \varepsilon \exp (2 M t) \\
& \geq f(t, x, w(t, x))+\int_{0}^{t} g(t, x, s, w(s, x)) d s+2 M \varepsilon \exp (2 M t) \\
& >f\left(t, x, w_{\varepsilon}(t, x)\right)+\int_{0}^{t} g\left(t, x, s, w_{\varepsilon}(s, x)\right) d s .
\end{aligned}
$$

Now using the strict inequality result, we have $w_{\varepsilon}(t, x)>v(t, x)$ for $t>0$. Letting $\varepsilon \rightarrow 0$, we have $w(t, x) \geq v(t, x)$ for $t>0$.

LEMMA 2.2. Assume that

(i) $g(t, x, s, u)$ is monotone nondecreasing in $u$ for each fixed $(t, x, s)$;

(ii) $v(t, x)$ satisfy

$$
\begin{gathered}
L v \leq f(t, x, v(t, x))+\int_{0}^{t} g(t, x, s, v(s, x)) d s \\
v(0, x)=u_{0}(x) \\
\left.v(t, x)\right|_{x \in \partial \Omega}=0
\end{gathered}
$$

and

$$
\begin{gathered}
r^{\prime}=h_{1}(t, r)+\int_{0}^{t} h_{2}(t, s, r) d s, \\
r(0)=\max \left\{\max _{x \in \Omega} u_{0}(x), 0\right\},
\end{gathered}
$$


where $h_{1}(t, r) \geq \max _{x \in \Omega} f(t, x, r)$ and $h_{2}(t, s, r) \geq \max _{x \in \Omega} g(t, x, s, r)$. Then we have $r(t) \geq v(t, x)$ on $\bar{Q}_{T}$.

Proof. Letting $V(t)=\max _{x \in \Omega} v(t, x)$, we have

$$
\begin{gathered}
V^{\prime} \leq f(t, x, V)+\int_{0}^{t} g(t, x, s, V(s)) d s \leq h_{1}(t, V)+\int_{0}^{t} h_{2}(t, s, V) d s, \\
V(0)=\max _{x \in \Omega} u_{0}(x) .
\end{gathered}
$$

This means that $V$ is a lower solution of $r$. Hence $r \geq V \geq v(t, x)$.

The proof can also be referred to Lemma 2.6 .2 on p. 72 of [8].

Next we develop the method of generalized quasilinearization for the parabolic integrodifferential equation (2.1). Using upper and lower solutions, we develop two monotone sequences that are solutions of linear parabolic integro-differential equations. We prove that the sequences converge uniformly and monotonically to the unique solution of the nonlinear parabolic integro-differential equation (2.1). Further, we prove that the rate of convergence is quadratic.

Theorem 2.1. Assume that

(i) $g(t, x, s, u)$ is monotone nondecreasing in $u$ for each fixed $(t, x, s) \in I \times \Omega \times I$, $f_{u u} \geq 0$.

(ii) $v_{0}(t, x)$ and $w_{0}(t, x)$ satisfy

$$
\begin{gathered}
L v_{0} \leq f\left(t, x, v_{0}(t, x)\right)+\int_{0}^{t} g\left(t, x, s, v_{0}(s, x)\right) d s \\
L w_{0} \geq f\left(t, x, w_{0}(t, x)\right)+\int_{0}^{t} g\left(t, x, s, w_{0}(s, x)\right) d s
\end{gathered}
$$

and

$$
\begin{aligned}
v_{0}(0, x) & \leq w_{0}(0, x), \\
\left.v_{0}(t, x)\right|_{x \in \partial \Omega} & \leq\left. w_{0}(t, x)\right|_{x \in \partial \Omega} .
\end{aligned}
$$

(iii) $f_{u}, g_{u} \in C^{\alpha, \frac{\alpha}{2}}[I \times \Omega]$, and $f_{u u}, g_{u u}$ exist and are continuous such that $f_{u u} \geq 0$. There exist functions $\phi(t, x, s, u)$ and $G(t, x, s, u)=\phi(t, x, s, u)+g(t, x, s, u)$ such that $G_{u u} \geq 0, \phi_{u u}>0$.

(iv) $G_{u}\left(t, x, s, u_{1}\right)-\phi_{u}\left(t, x, s, u_{2}\right) \geq 0$, for $v_{0} \leq u_{1} \leq u_{2} \leq w_{0}$.

Then there exist monotone sequences $\left\{v_{n}\right\}$ and $\left\{w_{n}\right\}$ that converge monotonically and quadratically to the unique solution of $(2.1)$.

Proof. Initially we construct the sequences $\left\{v_{n}\right\}$ and $\left\{w_{n}\right\}$. For that purpose let $v_{1}$ and $w_{1}$ be the solutions of the linear parabolic integro-differential equations with initial and boundary conditions as

$$
\begin{aligned}
& L v_{1}= f\left(t, x, v_{0}(t, x)\right)+f_{u}\left(t, x, v_{0}(t, x)\right)\left(v_{1}(t, x)-v_{0}(t, x)\right) \\
&+\int_{0}^{t}\left\{g\left(t, x, s, v_{0}(s, x)\right)+\left[G_{u}\left(t, x, s, v_{0}(s, x)\right)\right.\right. \\
&\left.\left.\quad-\phi_{u}\left(t, x, s, w_{0}(s, x)\right)\right]\left(v_{1}(s, x)-v_{0}(s, x)\right)\right\} d s,
\end{aligned}
$$




$$
\begin{gathered}
v_{1}(0, x)=u_{0}(x), \quad x \in \Omega, \\
\left.v_{1}(t, x)\right|_{x \in \partial \Omega}=h(t, x),
\end{gathered}
$$

and

$$
\begin{gathered}
L w_{1}=f\left(t, x, w_{0}(t, x)\right)+f_{u}\left(t, x, w_{0}(t, x)\right)\left(w_{1}(t, x)-w_{0}(t, x)\right) \\
+\int_{0}^{t}\left\{g\left(t, x, s, w_{0}(s, x)\right)+\left[G_{u}\left(t, x, s, v_{0}(s, x)\right)\right.\right. \\
\left.\left.-\phi_{u}\left(t, x, s, w_{0}(s, x)\right)\right]\left(w_{1}(s, x)-w_{0}(s, x)\right)\right\} d s \\
w_{1}(0, x)=u_{0}(x), \quad x \in \Omega \\
\left.w_{1}(t, x)\right|_{x \in \partial \Omega}=h(t, x) .
\end{gathered}
$$

From Theorem 3.3 in [3], it follows that $\left(v_{1}, w_{1}\right)$ exist and are unique. We can now prove that $v_{0} \leq v_{1} \leq w_{1} \leq w_{0}$ on $\bar{Q}_{T}$.

First we let $\eta=v_{0}-v_{1}$. Since $\eta(0, x)=0,\left.\eta(t, x)\right|_{x \in \partial \Omega}=0$, we have

$$
\begin{aligned}
L \eta= & L v_{0}-L v_{1} \\
\leq & f\left(t, x, v_{0}(t, x)\right)-\left[f\left(t, x, v_{0}(t, x)\right)+f_{u}\left(t, x, v_{0}(t, x)\right)\left(v_{1}(t, x)-v_{0}(t, x)\right)\right] \\
& +\int_{0}^{t} g\left(t, x, s, v_{0}(s, x)\right) d s-\int_{0}^{t}\left\{g\left(t, x, s, v_{0}(s, x)\right)\right. \\
& \left.+\left[G_{u}\left(t, x, s, v_{0}(s, x)\right)-\phi_{u}\left(t, x, s, w_{0}(s, x)\right)\right]\left(v_{1}(s, x)-v_{0}(s, x)\right)\right\} d s \\
\leq & f_{u}\left(t, x, v_{0}(t, x)\right) \eta+\int_{0}^{t}\left[G_{u}\left(t, x, s, v_{0}(s, x)\right)-\phi_{u}\left(t, x, s, w_{0}(s, x)\right)\right] \eta d s .
\end{aligned}
$$

Now applying Lemma 2.1, it follows that $\eta \leq 0$. This proves $v_{0} \leq v_{1}$ on $\bar{Q}_{T}$.

Similarly, we can prove $w_{1} \leq w_{0}$ on $\bar{Q}_{T}$. In order to prove $v_{1} \leq w_{1}$ on $\bar{Q}_{T}$, we shall first prove $v_{1} \leq w_{0}$.

Setting $\xi=v_{1}-w_{0}$, we have $\xi(0, x)=0,\left.\xi(t, x)\right|_{x \in \partial \Omega}=0$. Now it follows that

$$
\begin{aligned}
L \xi \leq & f\left(t, x, v_{0}(t, x)\right)+f_{u}\left(t, x, v_{0}(t, x)\right)\left(v_{1}(t, x)-v_{0}(t, x)\right) \\
+ & \int_{0}^{t}\left\{g\left(t, x, s, v_{0}(s, x)\right)+\left[G_{u}\left(t, x, s, v_{0}(s, x)\right)\right.\right. \\
& \left.\left.\quad-\phi_{u}\left(t, x, s, w_{0}(s, x)\right)\right]\left(v_{1}(s, x)-v_{0}(s, x)\right)\right\} d s \\
& -f\left(t, x, w_{0}(t, x)\right)-\int_{0}^{t} g\left(t, x, s, w_{0}(s, x)\right) d s .
\end{aligned}
$$

Since $G_{u u} \geq 0, \phi_{u u}>0$, and $f_{u u}>0$, we get

$$
\begin{aligned}
\int_{0}^{t} g\left(t, x, s, w_{0}(s, x)\right) & \geq \int_{0}^{t}\left\{g\left(t, x, s, v_{0}(s, x)\right)\right. \\
& +G_{u}\left(t, x, s, v_{0}(s, x)\right)\left(w_{0}(s, x)-v_{0}(s, x)\right) \\
& \left.-\left[\phi\left(t, x, s, w_{0}(s, x)\right)-\phi\left(t, x, s, v_{0}(s, x)\right)\right]\right\} d s,
\end{aligned}
$$

and

$$
\phi\left(t, x, s, w_{0}(s, x)\right)-\phi\left(t, x, s, v_{0}(s, x)\right) \leq \phi_{u}\left(t, x, s, w_{0}(s, x)\right)\left(w_{0}(s, x)-v_{0}(s, x)\right) .
$$


Also, we have

$$
f\left(t, x, v_{0}(t, x)\right)-f\left(t, x, w_{0}(t, x)\right) \leq f_{u}\left(t, x, v_{0}(t, x)\right)\left(v_{1}-w_{0}\right) .
$$

This yields

$$
L \xi \leq f_{u}\left(t, x, v_{0}(t, x)\right) \xi+\int_{0}^{t}\left[G_{u}\left(t, x, s, v_{0}(s, x)\right)-\phi_{u}\left(t, x, s, w_{0}(s, x)\right)\right] \xi(x, s) d s,
$$

and using Lemma 2.1, we have $\xi \leq 0$, i.e., $v_{1} \leq w_{0}$.

Similarly, we can prove $v_{0} \leq w_{1}$.

Now we prove $v_{1} \leq w_{1}$ on $\bar{Q}_{T}$. Setting $\alpha=v_{1}-w_{1}$, we get

$$
\begin{aligned}
L \alpha= & f\left(t, x, v_{0}(t, x)\right)+f_{u}\left(t, x, v_{0}(t, x)\right)\left(v_{1}(t, x)-v_{0}(t, x)\right) \\
& +\int_{0}^{t}\left\{g\left(t, x, s, v_{0}(s, x)\right)-g\left(t, x, s, w_{0}(s, x)\right)+\left[G_{u}\left(t, x, s, v_{0}(s, x)\right)\right.\right. \\
& \left.\left.\quad-\phi_{u}\left(t, x, s, w_{0}(s, x)\right)\right]\left(v_{1}(s, x)-v_{0}(s, x)-\left(w_{1}(s, x)-w_{0}(s, x)\right)\right)\right\} d s .
\end{aligned}
$$

Since $f_{u u} \geq 0$, we get

$$
f\left(t, x, v_{0}(t, x)\right)-f\left(t, x, w_{0}(t, x)\right) \leq-f_{u}\left(t, x, v_{0}(t, x)\right)\left(w_{0}(t, x)-v_{0}(t, x)\right) .
$$

Using this, we obtain

$$
\begin{aligned}
f\left(t, x, v_{0}(t, x)\right) & +f_{u}\left(t, x, v_{0}(t, x)\right)\left(v_{1}(t, x)-\right. \\
-\left[f\left(t, x, w_{0}(t, x)\right)+f_{u}(t, x, x)\right) & \left.\left.w_{0}(t, x)\right)\left(w_{1}(t, x)-w_{0}(t, x)\right)\right] \\
& \leq-f_{u}\left(t, x, v_{0}(t, x)\right)\left(w_{1}(t, x)-v_{1}(t, x)\right) .
\end{aligned}
$$

Since $G_{u u} \geq 0$, we also have

$$
\begin{aligned}
& \int_{0}^{t}\left\{g\left(t, x, s, v_{0}(s, x)\right)-g\left(t, x, s, w_{0}(s, x)\right)\right. \\
& \left.\quad+\left[G_{u}\left(t, x, s, v_{0}(s, x)\right)-\phi_{u}\left(t, x, s, w_{0}(s, x)\right)\right]\left(w_{0}(s, x)-v_{0}(s, x)\right)\right\} d s \leq 0 .
\end{aligned}
$$

From this, it easily follows that

$$
L \alpha \leq f_{u}\left(t, x, v_{0}(t, x)\right) \alpha+\int_{0}^{t}\left[G_{u}\left(t, x, s, v_{0}(s, x)\right)-\phi_{u}\left(t, x, s, w_{0}(s, x)\right)\right] \alpha d s .
$$

By comparison Lemma 2.1, this yields $\alpha \leq 0$. This implies $v_{1} \leq w_{1}$ on $\bar{Q}_{T}$.

Assume that for some $n>0$ we have

$$
v_{0} \leq v_{1} \leq \cdots \leq v_{n} \leq w_{n} \leq \cdots \leq w_{1} \leq w_{0}
$$

Certainly (2.2) is true for $n=1$.

Let $v_{n+1}$ and $w_{n+1}$ be the solutions of the following linear equations:

$$
\begin{aligned}
L v_{n+1}= & f\left(t, x, v_{n}(t, x)\right)+f_{u}\left(t, x, v_{n}(t, x)\right)\left(v_{n+1}(t, x)-v_{n}(t, x)\right) \\
+ & \int_{0}^{t}\left\{g\left(t, x, s, v_{n}(s, x)\right)+\left[G_{u}\left(t, x, s, v_{n}(s, x)\right)\right.\right. \\
& \left.\left.-\phi_{u}\left(t, x, s, w_{n}(s, x)\right)\right]\left(v_{n+1}(s, x)-v_{n}(s, x)\right)\right\} d s,
\end{aligned}
$$




$$
\begin{gathered}
v_{n+1}(0, x)=u_{0}(x), \quad x \in \Omega, \\
\left.v_{n+1}(t, x)\right|_{x \in \partial \Omega}=h(t, x),
\end{gathered}
$$

and

$$
\begin{gathered}
L w_{n+1}=f\left(t, x, w_{n}(t, x)\right)+f_{u}\left(t, x, w_{n}(t, x)\right)\left(w_{n+1}(t, x)-w_{n}(t, x)\right) \\
+\int_{0}^{t}\left\{g\left(t, x, s, w_{n}(s, x)\right)+\left[G_{u}\left(t, x, s, v_{n}(s, x)\right)\right.\right. \\
\left.\left.-\phi_{u}\left(t, x, s, w_{n}(s, x)\right)\right]\left(w_{n+1}(s, x)-w_{n}(s, x)\right)\right\} d s, \\
w_{n+1}(0, x)=u_{0}(x), \quad x \in \Omega, \\
\left.w_{n+1}(t, x)\right|_{x \in \partial \Omega}=h(t, x) .
\end{gathered}
$$

We assume that (2.2) is true for some $n$, and we prove that $v_{n} \leq v_{n+1} \leq w_{n+1} \leq w_{n}$. This proves (2.2) is true for $n=n+1$ also.

First we show that $v_{n} \leq v_{n+1}$, by letting $\alpha=v_{n+1}-v_{n}$. Since

$$
\begin{aligned}
& \int_{0}^{t} g\left(t, x, s, v_{n}(s, x)\right) d s \\
& \geq \int_{0}^{t}\left\{g\left(t, x, s, v_{n-1}(s, x)\right)+G_{u}\left(t, x, s, v_{n-1}(s, x)\left(v_{n}(s, x)-v_{n-1}(s, x)\right)\right)\right. \\
& \left.\quad-\left[\phi\left(t, x, s, v_{n}(s, x)\right)-\phi\left(t, x, s, v_{n-1}(s, x)\right)\right]\right\} d s \\
& \geq \int_{0}^{t}\left\{g\left(t, x, s, v_{n-1}(s, x)\right)\right. \\
& \left.\quad+\left[G_{u}\left(t, x, s, v_{n-1}(s, x)\right)-\phi_{u}\left(t, x, s, v_{n-1}(s, x)\right)\right]\left(v_{n}(s, x)-v_{n-1}(s, x)\right)\right\} d s \\
& \geq \int_{0}^{t}\left\{g\left(t, x, s, v_{n-1}(s, x)\right)\right. \\
& \left.\quad+\left[G_{u}\left(t, x, s, v_{n-1}(s, x)\right)-\phi_{u}\left(t, x, s, w_{n-1}(s, x)\right)\right]\left(v_{n}(s, x)-v_{n-1}(s, x)\right)\right\} d s,
\end{aligned}
$$

and

$$
f\left(t, x, v_{n}(t, x)\right) \geq f\left(t, x, v_{n-1}(t, x)\right)+f_{u}\left(t, x, v_{n-1}(t, x)\right)\left(v_{n}(t, x)-v_{n-1}(t, x)\right),
$$

it easily follows that

$$
\begin{aligned}
L \alpha= & f\left(t, x, v_{n}(t, x)\right)+f_{u}\left(t, x, v_{n}(t, x)\right)\left(v_{n+1}(t, x)-v_{n}(t, x)\right) \\
& +\int_{0}^{t}\left\{g\left(t, x, s, v_{n}(s, x)\right)+\left[G_{u}\left(t, x, s, v_{n}(s, x)\right)\right.\right. \\
& \left.\left.\quad-\phi_{u}\left(t, x, s, w_{n}(s, x)\right)\right]\left(v_{n+1}(s, x)-v_{n}(s, x)\right)\right\} d s \\
& -\left[f\left(t, x, v_{n-1}(t, x)\right)+f_{u}\left(t, x, v_{n-1}(t, x)\right)\left(v_{n}(t, x)-v_{n-1}(t, x)\right)\right. \\
& +\int_{0}^{t}\left\{g\left(t, x, s, v_{n-1}(s, x)\right)+\left[G_{u}\left(t, x, s, v_{n-1}(s, x)\right)\right.\right. \\
& \left.\left.\left.\quad-\phi_{u}\left(t, x, s, w_{n-1}(s, x)\right)\right]\left(v_{n}(s, x)-v_{n-1}(s, x)\right)\right\} d s\right] \\
\geq & f_{u}\left(t, x, v_{n}(t, x)\right) \alpha+\int_{0}^{t}\left[G_{u}\left(t, x, s, v_{n}(s, x)\right)-\phi_{u}\left(t, x, s, w_{n}(s, x)\right)\right] \alpha d s .
\end{aligned}
$$


By the comparison Lemma 2.1, it follows that $v_{n} \leq v_{n+1}$ on $\bar{Q}_{T}$.

Next we show that $w_{n+1} \leq w_{n}$. Letting $\beta=w_{n+1}-w_{n}$, we have

$$
\begin{aligned}
L \beta= & f\left(t, x, w_{n}(t, x)\right)+f_{u}\left(t, x, w_{n}(t, x)\right)\left(w_{n+1}(t, x)-w_{n}(t, x)\right) \\
& +\int_{0}^{t}\left\{g\left(t, x, s, w_{n}(s, x)\right)+\left[G_{u}\left(t, x, s, v_{n}(s, x)\right)\right.\right. \\
& \left.\left.\quad-\phi_{u}\left(t, x, s, w_{n}(s, x)\right)\right]\left(w_{n+1}(s, x)-w_{n}(s, x)\right)\right\} d s \\
& -\left[f\left(t, x, w_{n-1}(t, x)\right)+f_{u}\left(t, x, w_{n-1}(t, x)\right)\left(w_{n}(t, x)-w_{n-1}(t, x)\right)\right. \\
+ & \int_{0}^{t}\left\{g\left(t, x, s, w_{n-1}(s, x)\right)+\left[G_{u}\left(t, x, s, v_{n-1}(s, x)\right)\right.\right. \\
& \left.\left.\left.\quad-\phi_{u}\left(t, x, s, w_{n-1}\right)(s, x)\right)\right]\left(w_{n}(s, x)-w_{n-1}(s, x)\right)\right\} d s .
\end{aligned}
$$

Since $G_{u u} \geq 0$ and $\phi_{u u} \geq 0$, we also have

$$
\begin{aligned}
& \int_{0}^{t}\left\{g\left(t, x, s, w_{n}(s, x)\right)-g\left(t, x, s, w_{n-1}(s, x)\right) d s\right. \\
& \left.\quad \leq \int_{0}^{t}\left[G_{u}\left(t, x, s, v_{n-1}(s, x)\right)-\phi_{u}\left(t, x, s, w_{n-1}(s, x)\right)\right]\left(w_{n}(s, x)-w_{n-1}(s, x)\right)\right\} d s .
\end{aligned}
$$

Since $w_{n} \leq w_{n-1}$ and $f_{u u} \geq 0$, by our assumption we have

$$
f\left(t, x, w_{n}(t, x)\right) \leq f\left(t, x, w_{n-1}(t, x)\right)+f_{u}\left(t, x, w_{n-1}(t, x)\right)\left(w_{n}(t, x)-w_{n-1}(t, x)\right) .
$$

Hence it follows that

$$
L \beta \leq f_{u}\left(t, x, w_{n}(t, x)\right) \beta+\int_{0}^{t}\left[G_{u}\left(t, x, s, v_{n-1}(s, x)\right)-\phi_{u}\left(t, x, s, w_{n-1}(s, x)\right)\right] \beta d s .
$$

Again using the comparison Lemma 2.1, we obtain that $w_{n+1} \leq w_{n}$.

On the same lines as we proved $v_{1} \leq w_{1}$ on $\bar{Q}_{T}$, we can also prove $v_{n+1} \leq w_{n+1}$.

We have established (2.2) for $n=1$. Now using mathematical induction, it follows that

$$
v_{0} \leq v_{1} \leq \cdots \leq v_{n} \leq v_{n+1} \leq w_{n+1} \leq w_{n} \leq \cdots \leq w_{1} \leq w_{0}, \quad \text { for all } n .
$$

Let $\bar{v}(t, x)=\lim _{n \rightarrow \infty} v_{n}(t, x)$ and $\underline{w}(t, x)=\lim _{n \rightarrow \infty} w_{n}(t, x)$. Since $\left\{v_{n}\right\}$ and $\left\{w_{n}\right\}$ possess the monotone property, it ensures that $\bar{v}(t, x)$ and $\underline{w}(t, x)$ exist. By the same method as in [8], it is easy to show that $\bar{v}(t, x)=\underline{w}(t, x)$ and is the unique solution of (2.1).

We claim that the convergence of $v_{n}$ is uniform. Assume that the claim is false. Then for some $\varepsilon_{0}>0$, and for any $i$, there exist $n_{i}>i$ and $\left(t_{n_{i}}, x_{n_{i}}\right) \in \bar{Q}_{T}$ such that

$$
\left|v_{n_{i}}\left(t_{n_{i}}, x_{n_{i}}\right)-u\left(t_{n_{i}}, x_{n_{i}}\right)\right|>\varepsilon_{0} .
$$

We choose $i>n_{i-1}$ so that we can construct a sequence $\left\{\left(t_{n_{i}}, x_{n_{i}}\right)\right\}$. Since $\bar{Q}_{T}$ is compact, there exists a subsequence $\left\{\left(t_{n_{j}}, x_{n_{j}}\right)\right\}$ such that $\left\{\left(t_{n_{j}}, x_{n_{j}}\right)\right\}$ has a limit $(\bar{t}, \bar{x})$. Since $v_{n}$ and $u$ are uniformly continuous, it follows from (2.3) that

$$
\varepsilon_{0} \leq \lim _{n_{j} \rightarrow \infty}\left|v_{n_{j}}\left(t_{n_{j}}, x_{n_{j}}\right)-u\left(t_{n_{j}}, x_{n_{j}}\right)\right|=|\bar{v}(\bar{t}, \bar{x})-u(\bar{t}, \bar{x})|=0
$$

which is a contradiction. Similarly we can prove that $w_{n}$ converges uniformly to $\underline{w}(t, x)=$ $u(t, x)$, the unique solution of $(2.1)$. 
Finally, we show that the convergence is quadratic. For that, let $p_{n+1}=u(t, x)-v_{n+1}$ and $q_{n+1}=w_{n+1}-u(t, x)$. It follows that

$$
\begin{aligned}
L p_{n+1}= & f(t, x, u(t, x))-\left[f\left(t, x, v_{n}(t, x)\right)+f_{u}\left(t, x, v_{n}(t, x)\right)\left(v_{n+1}(t, x)-v_{n}(t, x)\right)\right] \\
& +\int_{0}^{t} g(t, x, s, u(s, x)) d s-\int_{0}^{t}\left\{g\left(t, x, s, v_{n}(s, x)\right)+\left[G_{u}\left(t, x, s, v_{n}(s, x)\right)\right.\right. \\
= & f_{u}\left(t, x, v_{n}(t, x)\right)\left(u(t, x)-v_{n}(t, x)\right)+f_{u u}(t, x, \xi)\left(u(t, x)-v_{n}(t, x)\right)^{2} \\
& \left.-f_{u}\left(t, x, v_{n}(t, x)\right)\left(v_{n+1}(t, x)-v_{n}(t, x)\right)\right\} d s \\
& +\int_{0}^{t}\left[g(t, x, s, u(s, x))-g\left(t, x, s, v_{n}(s, x)\right)\right] d s \\
& -\int_{0}^{t}\left\{[ G _ { u } ( t , x , s , v _ { n } ( s , x ) ) - \phi _ { u } ( t , x , s , w _ { n } ( s , x ) ) ] \left(v_{n+1}(s, x)-u(t, x)\right.\right. \\
= & f_{u}\left(t, x, v_{n}(t, x)\right)\left(u(t, x)-v_{n+1}(t, x)\right)+f_{u u}(t, x, \xi)\left(u(t, x)-v_{n}(t, x)\right)^{2} \\
& +\int_{0}^{t} g_{u}(t, x, s, \mu) p_{n} d s+\int_{0}^{t}\left[G_{u}\left(t, x, s, v_{n}(s, x)\right)-\phi_{u}\left(t, x, s, w_{n}(s, x)\right)\right] p_{n+1} d s \\
& -\int_{0}^{t}\left[G_{u}\left(t, x, s, v_{n}(s, x)\right)-\phi_{u}\left(t, x, s, w_{n}(s, x)\right)\right] p_{n} d s \\
\leq & f_{u}\left(t, x, v_{n}(t, x)\right) p_{n+1}+f_{u u}(t, x, \xi) p_{n}^{2} \\
& +\int_{0}^{t} g_{u}\left(t, x, s, w_{n}(s, x)\right) p_{n} d s \\
& +\int_{0}^{t}\left[G_{u}\left(t, x, s, v_{n}(s, x)\right)-\phi_{u}\left(t, x, s, w_{n}(s, x)\right)\right] p_{n+1} d s \\
\leq & M_{1} p_{n+1}+M_{2} p_{n}^{2}+\int_{0}^{t}\left[M_{3} p_{n}^{2}+\frac{1}{2} M_{3}\left(p_{n}^{2}+q_{n}^{2}\right)+M_{4} p_{n+1}\right] d s, \\
\leq & f_{u}\left(t, x, v_{n}(t, x)\right) p_{n+1}+f_{u u}(t, x, \xi) p_{n}^{2} \\
& +\int_{0}^{t} G_{u u}\left(t, x, s, \mu_{1}\right)\left(p_{n}+q_{n}\right) p_{n} d s+\int_{0}^{t} g_{u}\left(t, x, s, v_{n}(s, x)\right) p_{n+1} d s \\
& \\
& \\
& \\
&
\end{aligned}
$$

where $\left|f_{u}\right| \leq M_{1},\left|f_{u u}\right| \leq M_{2},\left|G_{u u}\right| \leq M_{3},\left|g_{u}\right| \leq M_{4},\left|g_{u u}\right| \leq M_{5}$.

Clearly $p_{n+1}(0, x)=0,\left.p_{n+1}(t, x)\right|_{x \in \partial \Omega}=0$.

Let $r(t)$ be the solution of the related IVP for a linear integro-differential equation. Then we have

$$
\begin{aligned}
r^{\prime}(t) & =M_{1} r(t)+M_{4} \int_{0}^{t} r(s) d s+\left(M_{2}+\frac{3}{2} M_{3} T\right) \max _{x \in \Omega, 0<t<T} p_{n}^{2}+\frac{1}{2} M_{3} T \max _{x \in \Omega, 0<t<T} q_{n}^{2}, \\
r(0) & =0 .
\end{aligned}
$$


We obtain the following estimate for $r(t)$ :

$$
r(t) \leq \frac{2 \exp \left(M_{1} T\right)}{\sqrt{M_{1}^{2}+4 M_{4}}}\left[\left(M_{2}+\frac{3}{2} M_{3} T\right) \max _{x \in \Omega, 0<t<T} p_{n}^{2}+\frac{1}{2} M_{3} T \max _{x \in \Omega, 0<t<T} q_{n}^{2}\right] .
$$

It is easy to see that

$$
\int_{0}^{t}\left[M_{3} p_{n}^{2}+\frac{1}{2} M_{3}\left(p_{n}^{2}+q_{n}^{2}\right)\right] d s \leq \frac{3}{2} M_{3} T \max _{x \in \Omega, 0<t<T} p_{n}^{2}+\frac{1}{2} M_{3} T \max _{x \in \Omega, 0<t<T} q_{n}^{2} .
$$

Using Lemma 2.2 we have $p_{n+1}(t, x) \leq r(t)$. Therefore

$$
\max _{x \in \Omega, 0<t<T} p_{n+1} \leq \frac{2 \exp \left(M_{1} T\right)}{\sqrt{M_{1}^{2}+4 M_{4}}}\left[\left(M_{2}+\frac{3}{2} M_{3} T\right) \max _{x \in \Omega, 0<t<T} p_{n}^{2}+\frac{1}{2} M_{3} T \max _{x \in \Omega, 0<t<T} q_{n}^{2}\right] .
$$

Similarly,

$$
\begin{aligned}
\max _{x \in \Omega, 0<t<T} q_{n+1} \leq \frac{2 \exp \left(M_{1} T\right)}{\sqrt{M_{1}^{2}+4 M_{4}}}\left[\left(M_{2}+\frac{3}{2} T M_{3}+T M_{5}\right)\right. & \max _{x \in \Omega, 0<t<T} q_{n}^{2} \\
& \left.+\frac{1}{2} M_{3} T \max _{x \in \Omega, 0<t<T} p_{n}^{2}\right] .
\end{aligned}
$$

Hence, the sequences $\left\{v_{n}\right\}$ and $\left\{w_{n}\right\}$ converge quadratically to $u(t, x)$, the unique solution of (2.1). The proof is complete.

REMARK 2.1. Actually, we do not need to assume $f_{u u} \geq 0$. It is enough if there exists a function $\psi(t, x, s, u)$ such that $F(t, x, s, u)=\psi(t, x, s, u)+f(t, x, s, u)$ and $F_{u u} \geq$ $0, \psi_{u u}>0$. It is easy to see that we can always construct a $\psi(t, x, s, u)$ that satisfies the above inequalities.

3. Numerical examples. In this section, we apply the generalized quasilinearization method to the following problem, which models the propagation of a voltage pulse through a nerve axon, often referred to as the Fitzhugh-Nagumo equation [8],

$$
u_{t}-u_{x x}=u(u-\theta)(1-u)+b \int_{0}^{t} u(s, x) d s, \quad 0<x, t<1 .
$$

Here, we choose $\theta=0.5$ and $b=0$, with the initial boundary conditions as

$$
\begin{array}{ll}
u(0, x)=\sin (\pi x), & 0<x<1, \\
u(0, t)=u(1, t)=0, & 0<t<1 .
\end{array}
$$

Let $\psi(t, x, u)=u^{3}+\theta u$. Then it is easy to see that $f+\psi$ is convex.

For each step of the iteration in the generalized quasilinearization method, we have the following linear IBVPs:

$$
\begin{gathered}
\frac{\partial}{\partial t} v^{(k+1)}-\frac{\partial^{2}}{\partial x^{2}} v^{(k+1)}=v^{(k)}\left(v^{(k)}-\theta\right)\left(1-v^{(k)}\right) \\
+\left(2(1+\theta) w^{(k)}-\theta-3\left(v^{(k)}\right)^{2}\right)\left(v^{(k+1)}-v^{(k)}\right)+\int_{0}^{t} v^{(k+1)} d s \\
v^{(k+1)}(0, x)=\sin (\pi x), \quad 0<x<1, \\
\left.v^{(k+1)}(t, x)\right|_{x=0,1}=0,
\end{gathered}
$$


and

$$
\begin{gathered}
\frac{\partial}{\partial t} w^{(k+1)}-\frac{\partial^{2}}{\partial x^{2}} w^{(k+1)}=w^{(k)}\left(w^{(k)}-\theta\right)\left(1-w^{(k)}\right) \\
+\left(2(1+\theta) w^{(k)}-\theta-3\left(v^{(k)}\right)^{2}\right)\left(w^{(k+1)}-w^{(k)}\right)+b \int_{0}^{t} w^{(k+1)} d s, \\
w^{(k+1)}(0, x)=\sin (\pi x), \quad 0<x<1, \\
\left.w^{(k+1)}(t, x)\right|_{x=0,1}=0 .
\end{gathered}
$$

The solutions of (3.2) and (3.3) can be obtained by the implicit method. The final finite difference equations are

$$
\begin{aligned}
& \frac{v^{(k+1)}(l+1, i)-v^{(k+1)}(l, i)}{\Delta t} \\
& \quad-\frac{v^{(k+1)}(l+1, i-1)-2 v^{(k+1)}(l, i)+v^{(k+1)}(l, i+1)}{(\Delta x)^{2}} \\
& =v^{(k)}(l+1, i)\left(v^{(k)}(l+1, i)-\theta\right)\left(1-v^{(k)}(l+1, i)\right. \\
& \quad+\left(2(1+\theta) w^{(k)}(l+1, i)-\theta-3\left(v^{(k)}(l+1, i)\right)^{2}\right)\left(v^{(k+1)}(l+1, i)-v^{(k)}(l+1, i)\right) \\
& \quad+\left(b \sum_{m=1}^{l} v^{(k+1)}(m, i)+\frac{1}{2} v^{(k+1)}(l+1, i)+\frac{1}{2} v^{(k+1)}(0, i)\right) \Delta t,
\end{aligned}
$$

and

$$
\begin{aligned}
& \frac{w^{(k+1)}(l+1, i)-w^{(k+1)}(l, i)}{\Delta t} \\
& \quad-\frac{w^{(k+1)}(l+1, i-1)-2 w^{(k+1)}(l, i)+w^{(k+1)}(l, i+1)}{(\Delta x)^{2}} \\
& =w^{(k)}(l+1, i)\left(w^{(k)}(l+1, i)-\theta\right)\left(1-w^{(k)}(l+1, i)\right. \\
& \quad+\left(2(1+\theta) w^{(k)}(l+1, i)-\theta-3\left(v^{(k)}(l+1, i)\right)^{2}\right)\left(w^{(k+1)}(l+1, i)-w^{(k)}(l+1, i)\right) \\
& \quad+\left(b \sum_{m=1}^{l} w^{(k+1)}(m, i)+\frac{1}{2} w^{(k+1)}(l+1, i)+\frac{1}{2} w^{(k+1)}(0, i)\right) \Delta t
\end{aligned}
$$

where $v^{(k)}(l, i)=v^{(k)}(l \Delta t, i \Delta x)$.

We take $L=400, I=20, \Delta t=1 / L, \Delta x=1 / I$, and $v_{0}=0, w_{0}=\sin (\pi x)$ as lower and upper solutions, respectively. We use the LU decomposition, and we stop the iteration when the difference of lower and upper solutions is small enough. Actually, we take the difference $\varepsilon=10^{-5}$. Figures $3.1,3.2$, and 3.3 show the changes of lower solution, upper solution, and the difference between them. 

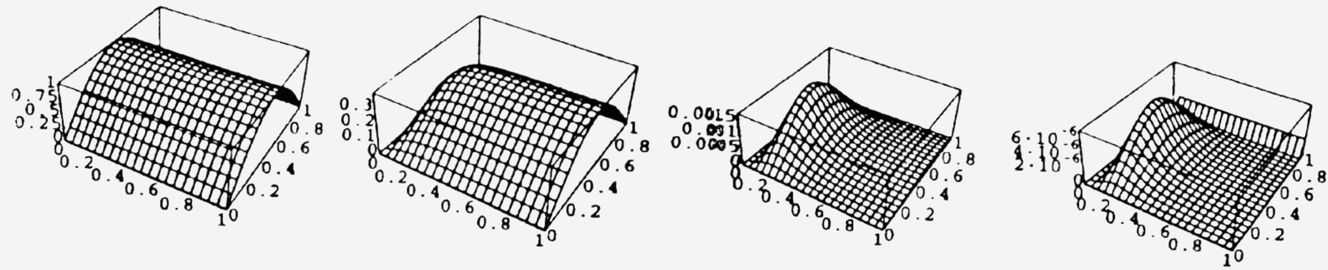

FIG. 3.1. The distance surface between lower and upper solutions
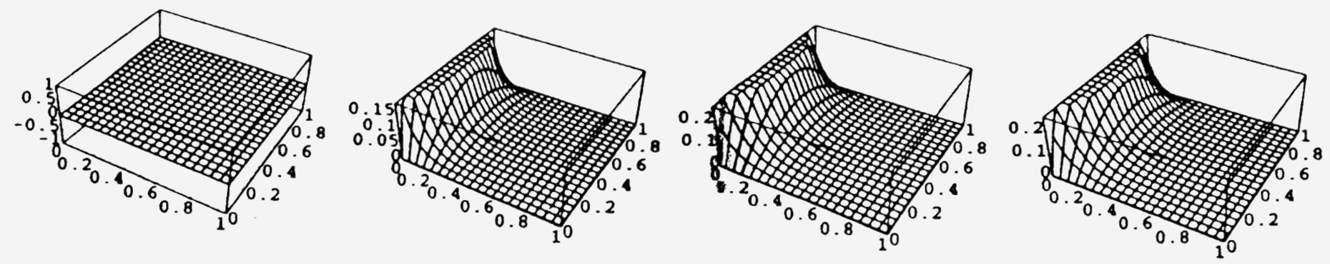

FIG. 3.2. The lower solution for the $0,1,3,5$ iteration

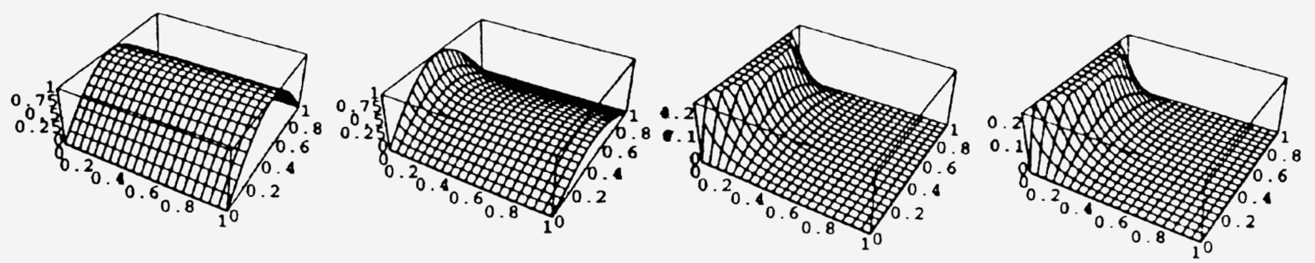

FIG. 3.3. The upper solution for the $0,1,3,5$ iteration

\section{REFERENCES}

[1] R. Bellman, Methods of Nonlinear Analysis, Vol. II, Academic Press, New York, 1973

[2] R. Bellman and R. Kalaba, Quasilinearization and Nonlinear Boundary Value Problems, American Elsevier, New York, 1965

[3] J. Cannon and Y. P. Lin, Smooth solutions for an integro-differential equation of parabolic type, Differential and Integral Equations 2, 111-121 (1989)

[4] G. S. Ladde, V. Lakshmikantham, and A. S. Vatsala, Monotone Iterative Techniques for Nonlinear Differential Equations, Pitman, Boston, 1985

[5] V. Lakshmikantham and S. Leela, Differential and Integral Inequalities, vol. VII, Academic Press, New York, 1968

[6] V. Lakshmikantham and M. Rama Mohana Rao, Theory of Integro-Differential Equations, Stability and Control: Theory, Methods and Applications, Volume 1, Gordon and Breach Science Publishers, Lausanne, Switzerland, 1995

[7] V. Lakshmikantham and A. S. Vatsala, Generalized quasilinearization for nonlinear problems, Mathematics and its Applications 440, Kluwer Academic Publishers, Dordrecht, 1998

[8] C. V. Pao, Nonlinear Parabolic and Elliptic Equations, Plenum Press, New York, 1992

[9] S. G. Deo and C. McGloin Knoll, Further extension of the method of quasi-linearization to integrodifferential equations, International Journal of Nonlinear Differential Equations: Theory, Methods, and Applications, Vol. 3, 1997, pp. 91-103

[10] A. S. Vatsala, Generalized quasilinearization and reaction diffusion equations, Nonlinear Times and Digest 1, 211-220 (1994)

[11] D. Stutson and A. S. Vatsala, Quadratic and semi-quadratic convergence of IVP, Neural, Parallel and Scientific Computations 3, 235-248 (1995) 